\title{
FONEMAS SEGMENTALES DEL 'LUDAR', LENGUA HABLADA POR UN GRUPO GITANO DE ARGENTINA ${ }^{1}$
}

\author{
Gastón Salamanca
}

\begin{abstract}
RESUMEN
Este artículo presenta los fonemas segmentales del ludar hablado en Argentina. Además, incluye una breve introducción etnográfica de los gitanos, una referencia a estudios anteriores, algunos detalles del marco teórico empleado y un cuento en transcripción fonémica.
\end{abstract}

\begin{abstract}
This paper presents the segmental phonemes of the Argentinian Ludar, a dialect spoken by gypsies. It also includes an ethnographic introduction of this group, and some details about the theoretical frame applied in this study. Finally there is a tale with its phonemic transcription.
\end{abstract}

\section{Introducción}

Esta presentación se centra en el nivel fonológico. Su propósito es describir una lengua en particular: el ludar. Ludar es el nombre que utiliza un grupo de gitanos para denominarse a sí mismo y a su lengua. Este grupo se encuentra en diversos países de Europa y América ${ }^{2}$. El foco de este artículo lo constituye la variante hablada por los gitanos ludar de Buenos Aires, Argentina.

\section{Introducción etnográfica al pueblo gitano ${ }^{3}$}

\subsection{Gitanos en el mundo}

\subsubsection{Origen y migraciones}

Aunque se han elaborado numerosas teorías que explican el origen de los gitanos, hoy se tiene por un hecho plenamente demostrado que éstos provienen del norte de la India. De este subcontinente habrían emigrado alrededor del año mil después de Cristo (Hancock 1993).

La fecha de ingreso de los gitanos al continente europeo se estima alrededor de 1400. Migraciones masivas al continente americano se producen a mediados del siglo XIX (Vaux de F. 1984). 


\subsubsection{Nomadismo}

Se pueden distinguir tres grandes grupos:

Gitanos nómades: Viajan en carpas o carromatos durante todos los meses del año. Gitanos seminómades: Viajan durante el verano y en invierno se establecen en casas. Gitanos sedentarizados: Se establecen de manera permanente en casas.

\subsubsection{Grupos}

La publicación Journal of the Gypsy Lore Society reconoce como grupos gitanos, entre otros, a aquellos que se autodenominan Churari, Grastari, Kalaidzi, Kalderash, Lovara, Ludar, Mashari, Pavees, Rom/Roma, Romungros, Rusuya, Sinti, Tsehari, Ursari (http://metro.turnpike.net/R/rtracy/ glsindex/who1. html, destacado mío. Extraído de la página web de Journal of the Gypsy Lore Society).

\subsubsection{La lengua de los gitanos}

Kenrick (1979: 110) describe de la siguiente manera la situación lingüística de los gitanos:

When the Romani (Gypsy) people left India....they took with them their language, and this language is still used by over five million persons, ranging from the settled Gypsies of Eastern Europe to the nomadic Kalderash of Western Europe and the Americas. In a few instances the spoken Romani language itself has died out, as with the Boyash in Romania, in others, it survives only as lexis, using the syntax, morphology of the host language (En van der Voort 1996: 2).

Desde el punto de vista lingüístico es posible distinguir, en consecuencia, tres grandes grupos de gitanos: a) aquellos que hablan algún dialecto del romaní (como los gitanos rom de Chile y Argentina); b) aquellos que mantienen sólo palabras de origen romaní (como el grupo caló en España) y c) aquellos que han perdido todo conocimiento del romaní (como el grupo ludar de Chile y Argentina).

\subsection{Gitanos en Argentina}

\subsubsection{Antecedentes generales}

En mi trabajo "Gitanos: Algunos antecedentes etnográficos”, mencioné las siguientes observaciones generales respecto de los gitanos de Argentina:

Procedencia: Aunque no se sabe la fecha exacta de ingreso de los gitanos a Argentina, se estima que ésta se produjo a mediados del siglo XIX. De acuerdo a nuestras observaciones realizadas in situ podemos afirmar que en este país se encuentran gitanos provenientes de Serbia, Rusia, Rumania, Grecia, Hungría y España.

Grupos: En Argentina, al igual que en Chile, existen dos grandes grupos de gitanos diferenciados lingüísticamente. Estos se denominan a sí mismos rom y ludar. El grupo mayoritario, y 
más conservador en cuanto a sus costumbres, es el grupo rom. Estos distinguen entre sí subgrupos, de acuerdo al lugar desde el cual emigraron. Así, por ejemplo, los gitanos rom de Argentina mencionan como "razas" distintas a los "húngaros", "rusos", "grecos", "machuanos", etc. El grupo ludar también distingue subgrupos de acuerdo al lugar desde el cual emigraron. Así, mencionan como grupos aparte a los "bosniacos", los "serbianes", etc.

La lengua: Los gitanos rom de Argentina llaman a su lengua romanés; los gitanos ludar la llaman ludar, limba luderiaste o rumano. Ludar es la denominación más frecuente.

La evidencia de que el grupo ludar y el grupo rom hablan dos lenguas distintas -y no meros dialectos de una misma lengua- proviene no sólo del nombre que éstos les dan, sino también de la imposibilidad de mutua comprensión cuando las hablan (la interacción entre ambos grupos se produce sólo en castellano) (1999: 113).

\subsubsection{Gitanos ludar de Argentina}

Considerando que el foco de este artículo lo constituye la fonología de la lengua hablada por el grupo gitano ludar, daré a continuación una descripción etnográfica más detallada ${ }^{4}$.

\subsubsection{Nomadismo}

Los gitanos ludar de Argentina se pueden clasificar en completamente nómades y sedentarizados. Los gitanos completamente nómades viven todo el año en carpas (๕erg). Estas se levantan sobre unos postes de madera denominados fúrka (postes centrales), puls (postes de los costados) y veriánd (postes horizontales). A diferencia de las carpas de los gitanos rom las cuales son muy coloridas externamente y están fabricadas en género- las carpas de los gitanos ludar están fabricadas en un material similar a la lona y son generalmente de un solo color (verde o azul).

Los gitanos nómades generalmente levantan sus carpas en sitios eriazos de precarias condiciones higiénicas.

Es interesante destacar que estos gitanos conservan más claramente los rasgos indúes propios de los primeros gitanos que emigraron desde la India (son delgados, de tez muy morena y cabello negro intenso).

Los gitanos ludar sedentarizados, por su parte, viven en casas propias en algunos barrios periféricos de la ciudad. El exterior de la casa es similar a la de cualquier vivienda occidental común. En el interior tampoco se observan rasgos distintivos de la cultura gitana. Contrasta esto último con el interior de las viviendas de los gitanos rom, en el cual destacan los objetos tradicionales (alfombras coloridas, samovaris, etc.).

Aunque el aspecto físico de los gitanos sedentarizados sugiere mayor grado de mezcla con el europeo occidental, hay algunos que mantienen la pigmentación oscura y el cabello negro intenso propios de los nómades.

\subsubsection{Oficios}

Los gitanos ludar se dedican a la compraventa de vehículos, a la administración de circos o pequeños parques de diversiones y a fotografiar niños en carritos tirados por caballos Pony.

Las mujeres se dedican a la quiromancia o a la lectura del tarot. 


\subsubsection{Educación formal}

La mayoría de los gitanos ludar de Argentina tienen una educación formal precaria. Aquellos que son nómades prácticamente no han asistido nunca a la escuela y son, en consecuencia, en su gran mayoría analfabetos. Los que se han establecido en casas tienden a valorar los beneficios de educarse, ya sea a través de la escuela argentina o en forma autodidacta.

\subsubsection{Bilingüismo}

Como he señalado en el punto anterior, los gitanos ludar llaman a su lengua ludar, ludariaste o rumano. El ludar se emplea como primera lengua entre los nómades. Los sedentarizados, en cambio, ostentan un bilingüismo subordinado al español.

Para concluir, señalaremos que, desde un punto de vista lingüístico-histórico, la pérdida del romaní por parte de los gitanos ludar (o boyás) parece estar íntimamente relacionada con la condición de esclavos a que fueron sometidos en Rumania durante el siglo XIX. Señala Grant:

Some groups which are historically and ethnically Romá have lost all knowledge of Romani: an example of these are the Boyas, descended from Romá who were house slaves during the period of serfdom in Rumanian from the fifteenth to the nineteenth century... (Grant 1994: 1)

Así, la situación lingüística de los gitanos ludar de Argentina, no parece ser muy distinta de la que se observa en otros países; por ejemplo, Hungría. Allí, como informa Réger,

...the so-called Boyash Gypsies speak certain dialects of the Rumanian language as their mother tongue. This may be the consequence of a language shift that ocurred in the Rumania area... (Réger 1995: 80).

\section{Estudios anteriores relativos a la fonología de la lengua de los gitanos en general y del grupo ludar en particular}

En Europa y Norteamérica se han publicado trabajos en los cuales se describe la fonología de la lengua hablada por los gitanos. Estos trabajos se contienen generalmente en gramáticas. Así, por ejemplo, se pueden citar Grammar of Vlax Romani (Hancock 1993), "Brief Grammar of Romani Language" (Rishi 1981), etc. Todos ellos se ocupan de la descripción de la lengua romaní.

Sobre la fonología de la lengua hablada por los gitanos de Sudamérica, en cambio, la cantidad de trabajos publicados es muy escasa. En efecto, de acuerdo con los datos de que disponemos, hasta el año 1994 sólo en Brasil se habían publicado algunos artículos en esta dirección (todos ellos relativos a la lengua de los gitanos rom). En este contexto, la "Descripción fonológica del /Romané/ de Chile" (González y Salamanca 1994) constituye un aporte pionero a los estudios de la lengua de los gitanos en nuestro continente. Este estudio, como se desprende de su título, tiene como foco la lengua hablada por el grupo rom. Sobre la fonología de la lengua hablada por el grupo ludar, hasta donde sabemos, no existe en el Cono Sur de América estudio lingüístico alguno. 


\section{Análisis del material}

El marco teórico escogido para el análisis del material es el propuesto en Pike (1947). La aplicación "paso a paso" de este modelo se encuentra en Echeverría (1964).

\subsection{Premisas para el análisis fonológico}

a. Un sonido tiende a ser modificado por el sonido adyacente.

Una secuencia de sonidos implica una secuencia de movimientos articulatorios. Estos se influyen mutuamente. De esto resulta una influencia de un sonido sobre otro.

Esto es lo que sucede en el ludar de Argentina con la contoide nasal velar [ṇ] la cual sólo ocurre ante $[\mathrm{g}]$ y $[\mathrm{k}]$, que son también velares:

$$
\begin{aligned}
& \text { [língure] } \quad \text { 'cuchara' } \\
& \text { [mụ̣káre] } \quad \text { 'comer' }
\end{aligned}
$$

b. Los sonidos de una lengua presentan tendencia a la simetría.

Las realizaciones de los fonemas /b/, /d/ y /g/ ([b] [b]), ([d] [a]) y ([g] [g]), respectivamente) del ludar de Argentina reflejan esta tendencia: las realizaciones oclusivas se dan en posición inicial y después de consonante nasal, mientras que las fricativas se dan en los demás contextos.

c. Series características de sonidos ejercen una presión estructural en la interpretación fonémica de segmentos o series fónicas sospechosas.

Así, por ejemplo, las semivocales y semiconsonantes son segmentos de interpretación relativa: pueden ser considerados como vocales o como consonantes.

Esta premisa nos lleva a interpretar (ij] [j]) ([u] [w]) del ludar de Argentina como realizaciones de los fonemas semiconsonánticos /j/ y /w/, respectivamente. Una de las razones es que en posición inicial absoluta no hay grupos vocálicos aparte de los que formarían estos dos segmentos (ver 4.4.2).

\subsection{Procedimientos analíticos y criterios de distribución}

Para la determinación del estatus fonémico de los fonos del ludar se agruparon los fonos del ludar en pares sospechosos y luego se determinó el tipo de distribución y el estatus fonémico que estos fonos manifestaban.

\subsubsection{Agrupación de los fonos en pares dudosos o sospechosos.}

Los pares sospechosos (pares de fonos articulatoriamente similares) fueron analizados teniendo presente el siguiente "prejuicio metodológico": mientras no se pruebe lo contrario, se les considerará realizaciones de unidades funcionales distintas. 
Pares dudosos en ludar son, por ejemplo, [s] y [z], de acuerdo con su similitud fonética: [s] fricativo, dorsoalveolar, sordo

[z] fricativo, dorsoalveolar, sonoro

\subsubsection{Determinación del tipo de distribución y estatus fonémico de los pares dudosos del ludar}

a. Distribución contrastante

Un par sospechoso está en distribución contrastante si ocurre en contextos idénticos o similares y los enunciados de que forman parte tienen distinto significado, o sea, si ocurren en un par mínimo o par submínimo. Dos fonos en distribución contrastante se considerarán miembros de distintos fonemas.

Ejemplo de contraste en contextos análogos:

\begin{tabular}{lll}
\multicolumn{1}{c}{$\mathrm{V}_{-}$} & \multicolumn{1}{c}{$\mathrm{V}$-\# } \\
[s] [saláte] 'ensalada' & [asá] 'asî' & [kaús] 'cucharón' \\
[z] [zar] 'azúcar' & [nozít] 'cordón' & [izóz] 'abajo'
\end{tabular}

Luego, $[\mathrm{s}] \mathrm{y}[\mathrm{z}]$ pertenecen a fonemas distintos: /s/ y /z/.

b. Distribución alternante o variación libre

Un par dudoso está en variación libre si al ocurrir en un contexto idéntico no se produce un cambio de significado. Dos fonos en variación libre se considerarán alófonos de un mismo fonema.

Las contoides [¥] ([fricativa, bilabial, áfona]) y [f] ([fricativa, labiodental, áfona) son un ejemplo de variación libre en el ludar de Argentina:

[fok] 'fuego'

[Fok] 'fuego'

Luego, [f] y [₹] son alófonos del mismo fonema /f/.

c. Distribución complementaria

Dos fonos articulatoriamente similares están en distribución complementaria si ocurren en contextos mutuamente excluyentes (donde ocurre uno no ocurre el otro y viceversa). Dos fonos en distribución complementaria se considerarán alófonos de un mismo fonema.

Veamos, por ejemplo, la distribución de [d] y [a]:

[daríngka] (nombre propio)

[datarás] 'pantalón' 
[kwáise] 'cola'

[pot] 'puente'

[doi] 'dos'

Se observa que [d] y [a] ocurren en contextos mutuamente excluyentes. Luego, [d] y [a] están en distribución complementaria y son, por lo tanto, miembros de un mismo fonema /d/.

\subsection{Determinación de los principales rasgos fonotácticos}

En esta parte del análisis se establecieron las posibilidades de combinación de los fonemas en la lengua ludar. Así, por ejemplo, se determinó que "V" es un tipo silábico posible en el ludar, mientras que una secuencia como "VVCCC" no lo es.

\section{Fonemas segmentales del ludar de Argentina}

Luego de observar la distribución de cada fono, se pudo determinar que el ludar de Argentina tiene 30 fonemas segmentales, de los cuales /i, e, a,, , a, o, u/ son vocales, /p, t, k, b, d, $\mathrm{g}, \mathrm{f}, \mathrm{v}, \mathrm{s}, \mathrm{z}, \ddot{\mathrm{s}}, \mathrm{z}, \mathrm{c}, \mathrm{z}, \mathrm{c}, \mathrm{j}, \mathrm{m}, \mathrm{n}, \mathrm{l}, \mathrm{r}, \overline{\mathrm{I}} /$ consonantes $\mathrm{y} / \mathrm{j}$, w/, semiconsonantes.

\subsection{Vocales}

Los fonemas vocálicos se realizan como vocoides egresivas sonoras, orales, silábicas.

\begin{tabular}{|c|c|c|}
\hline Fonema & Alófonos & Ejemplos \\
\hline$/ \mathbf{i} /$ & $\begin{array}{l}\text { [i] } \\
\text { alta, cerrada, anterior, } \\
\text { no redondeada. }\end{array}$ & $\begin{array}{l}\text { /imós/ [i.mós] ‘sucio' } \\
\text { /cirjáse/ [ţ̇i.rjá.se] 'cereza' } \\
\text { /purómpi/ [pu.róm.pi] 'maíz' }\end{array}$ \\
\hline /e/ & $\begin{array}{l}{[\mathrm{e}]} \\
\text { media, cerrada, anterior, } \\
\text { no redondeada. }\end{array}$ & $\begin{array}{l}\text { /estjále/ [es.tjá.le] 'estrella' } \\
\text { /ardéju/ [ar.té.ju] [ar.đé.yu]'ají' } \\
\text { /gargaúne/ [gar.छa.ú.ne] 'abeja' }\end{array}$ \\
\hline lil & $\begin{array}{l}\text { [i] } \\
\text { alta, cerrada, central, } \\
\text { no redondeada. }\end{array}$ & 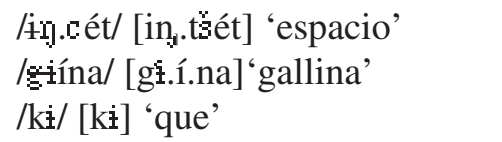 \\
\hline /a/ & $\begin{array}{l}{[\Xi]} \\
\text { media, cerrada, central, } \\
\text { no redondeada. }\end{array}$ & 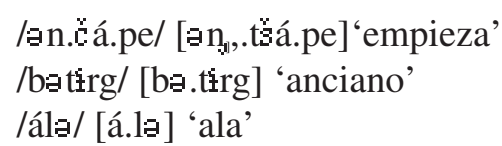 \\
\hline
\end{tabular}




\begin{tabular}{|c|c|c|}
\hline$/ \mathbf{a} /$ & $\begin{array}{l}\text { [a] } \\
\text { baja, abierta, central, } \\
\text { no redondeada. }\end{array}$ & 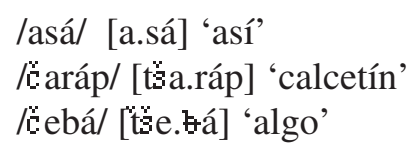 \\
\hline$/ 0 /$ & $\begin{array}{l}{[\mathrm{o}]} \\
\text { media, cerrada, posterior, } \\
\text { redondeada. }\end{array}$ & $\begin{array}{l}\text { /ólba/ [ól.もə] 'botella’ } \\
\text { /kokós/ [ko.kós] 'gallo’ } \\
\text { /jo/ [jo] [yo] ‘yo' }\end{array}$ \\
\hline$/ \mathrm{v}$ & $\begin{array}{l}{[\mathrm{u}]} \\
\text { alta, cerrada, posterior, } \\
\text { redondeada. }\end{array}$ & $\begin{array}{l}\text { /unsús/ [un.sús] 'arriba' } \\
\text { /búda/ [bú.Aa] 'carromato } \\
\text { /mácu/ [má.tsu] 'cuerda' }\end{array}$ \\
\hline
\end{tabular}

\subsection{Consonantes}

Los fonemas consonánticos se realizan como contoides egresivas no silábicas.

Fonema Alófonos

/p/ $\quad[\mathrm{p}]$

oclusiva, bilabial, sorda.

/b/ $\quad[\mathrm{b}]$

oclusiva, bilabial, sonora.

Ocurre en posición inicial

y después de consonante nasal.

[b]

fricativa, bilabial, sonora.

Ocurre en los demás contextos.

$/ \mathrm{t} / \quad[\mathrm{t}]$

oclusiva, postdental, sorda.

/d/ [d]

oclusiva, postdental, sonora.

Ocurre en posición inicial

absoluta y después de

consonante nasal.

[]

fricativa, postdental, sonora,

Ocurre en los otros contextos.
Ejémplos

/pidúrje/ [pi.đú.rje] 'bosque’

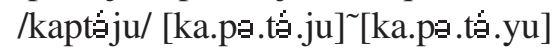
'cabecera'

/skjop/ [skjop] 'cojo'

/basíka/ [ba.sí.ka] 'ampolla'

/tambúrə/ [tam.bú.rə] 'guitarra’

/bába/ [bá.ba] 'abuela'

/tomovíla/ [to.mo.ví.la] 'automóvil' /zitín/ [zi.tín] 'aceite'

/git/ [git] 'garganta'

/dummazéw/ [dum.ma.zéw] 'Dios'

/dúnde/ [dúnin.de] 'dónde'

/ud/ [ü] 'mojado' 
$/ \mathbf{k} /$

[k] oclusiva, velar, sorda.

Ocurre ante vocal posterior y en posición final absoluta.

[k] oclusiva, postpalatal, sorda.

Ocurre en los demás contextos.

$/ g / \quad[\mathrm{g}]$

oclusiva, velar, sonora.

Ocurre ante vocal posterior:

-en posición inicial absoluta;

-después de consonante nasal.

[í⿴囗十

oclusiva, postpalatal, sonora.

Ocurre ante vocal no posterior:

-en posición inicial absoluta;

-después de consonante nasal.

[9]

fricativa, velar, sonora,

Ocurre ante vocal posterior,

en todo contexto, excepto

posición inicial y tras

consonante nasal.

[8]

fricativa, postpalatal, sonora.

Ocurre ante vocal anterior

en todo contexto, excepto

posición inicial y después de consonante nasal.

/f/

[f]

fricativa, labiodental, sorda. /kin/ [kin] 'cana'

/okólo/ [o.kó.lo] 'allá'

/nik/ [nik] 'chico'

/kisába/ [ki.sá.ła] 'cartera'

/gúra/ [gú.ra] 'boca'

/língure/ [líṇ.gu.re] 'cuchara'

/gjásta/ ['̣jás.ta] 'hielo’

/bogát/ [bo.gătt] 'rico'

/pagína/ [pa.sín.na] 'araña'

[F]

fricativa, bilabial, sorda.

Variación libre entre [f] y [₹]. 


\begin{tabular}{|c|c|c|}
\hline$/ \mathrm{v} /$ & $\begin{array}{l}\text { [v] } \\
\text { fricativa, labiodental, sonora. }\end{array}$ & $\begin{array}{l}\text { /vaselíe/ [va.se.lí.e] 'alfiler' } \\
\text { /nevún/ [ne.vún] 'tonto' } \\
\text { */_\#/ }\end{array}$ \\
\hline$/ \mathrm{s} /$ & $\begin{array}{l}\text { [s] } \\
\text { fricativa, dorsoalveolar, sorda. }\end{array}$ & $\begin{array}{l}\text { /sol/ [sol] 'alfombra' } \\
\text { /Énúsə/ [ți.nú.sə] 'ceniza' } \\
\text { /gros/ [gros] 'gordo' }\end{array}$ \\
\hline$|\mathbf{z}|$ & $\begin{array}{l}{[\mathrm{z}]} \\
\text { fricativa, dorsoalveolar, sonora. }\end{array}$ & $\begin{array}{l}\text { /zar/ [zar] 'azúcar' } \\
\text { /nozitt [no.zitt] 'cordón' } \\
\text { /ìzóz/ [立.zóz] 'abajo' }\end{array}$ \\
\hline / $/$ & $\begin{array}{l}{[\xi]} \\
\text { fricativa, alveopalatal, sorda. }\end{array}$ & 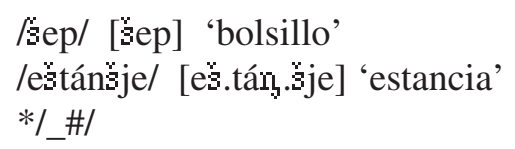 \\
\hline 晃/ & $\begin{array}{l}{[\tilde{z}]} \\
\text { fricativa, alveopalatal, sonora. }\end{array}$ & $\begin{array}{l}\text { /żinž.í/ [žiņ.ží] 'encía' } \\
\text { /ažúta/ [a.žú.ta] 'ayuda' } \\
\text { */_\#/ }\end{array}$ \\
\hline$/ \mathrm{c} /$ & $\begin{array}{l}\text { [ts] } \\
\text { africada, alveolar, sorda. }\end{array}$ & $\begin{array}{l}\text { /cagán/ [tsa.̇án] 'esposo' } \\
\text { /océru/ [o.tsé.ru] 'acero' } \\
\text { /burbec/ [bur.bets] 'gitano rom' }\end{array}$ \\
\hline$/ 2 /$ & $\begin{array}{l}{[\mathrm{dz}]} \\
\text { africada, alveolar, sonora. }\end{array}$ & $\begin{array}{l}\text { /z̧áce/ [dzá.ţ̌e] 'diez' } \\
\text { /fúze/ [fú.dze] 'huye' } \\
\text { */_\#/ }\end{array}$ \\
\hline 位/ & $\begin{array}{l}{[\mathrm{t}]} \\
\text { africada, alveopalatal, sorda. }\end{array}$ & 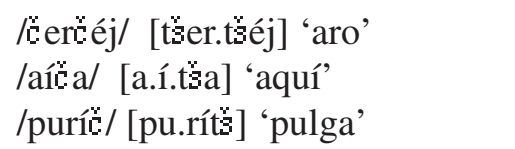 \\
\hline$/ \mathbf{j} /$ & $\begin{array}{l}{[\mathrm{d} z ̌ x]} \\
\text { africada, alveopalatal, sonora. }\end{array}$ & $\begin{array}{l}\text { /jinér/ [dži.nér] 'yerno' } \\
\text { /palậ̣áne/ [pa.la.džá.ne] 'tomate' } \\
\text { */_\#/ }\end{array}$ \\
\hline$/ \mathrm{m} /$ & $\begin{array}{l}{[\mathrm{m}]} \\
\text { nasal, bilabial, sonora. }\end{array}$ & $\begin{array}{l}\text { /mustáce/ [mus.tá.tse] 'bigote' } \\
\text { /lumanárja/ [lu.ma.ná.rja] 'vela' } \\
\text { /grom/ [grom] 'trueno' }\end{array}$ \\
\hline$/ \mathbf{n} /$ & $\begin{array}{l}{[\mathrm{n}]} \\
\text { nasal, alveolar, sonora. } \\
\text { Ocurre ante vocal y ante } \\
\text { consonante alveolar. }\end{array}$ & $\begin{array}{l}\text { /nu/ [nu] 'no' } \\
\text { /ốinúse/ [ţ̌i.nú.se] 'ceniza' } \\
\text { /fistán/ [fis.tán] 'falda' }\end{array}$ \\
\hline
\end{tabular}


[n]] /mìndru/ [mìng.dru] 'bonito'

nasal, postdental, sonora.

Ocurre ante consonante postdental.

$[\overline{\mathrm{I}}]$

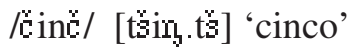

nasal, alveopalatalizada, sonora.

Ocurre ante consonante alveopalatal.

[ị] /inklisáste/ [ił̣̂.Kli.sás.te] 'enciende’ nasal, postpalatal, sonora. Ocurre ante consonante postpalatal

[ᄁ]] /munkáre/ [mự̣.ká.re] 'comer' nasal, velar, sonora. Ocurre ante consonante velar.

\begin{tabular}{|c|c|c|}
\hline$/ \mathbf{l} /$ & $\begin{array}{l}{[1]} \\
\text { lateral, alveolar, sonora. }\end{array}$ & $\begin{array}{l}\text { /láwte/ [lánٍ.te] 'leche' } \\
\text { /kopilás/ [ko.pi.lás] 'bebé' } \\
\text { /kal/ [kal] 'caballo' }\end{array}$ \\
\hline$/ \mathbf{r} /$ & $\begin{array}{l}{[\mathrm{r}]} \\
\text { vibrante simple, alveolar, } \\
\text { sonora. }\end{array}$ & $\begin{array}{l}\text { */\#_/ } \\
\text { /sturój/ [stu.roị] 'ajo' } \\
\text { /amár/ [a.mar] 'amargo' }\end{array}$ \\
\hline$/ \mathbf{I}$ & $\begin{array}{l}{[\bar{I}]} \\
\text { vibrante múltiple, alveolar, } \\
\text { sonora. }\end{array}$ & $\begin{array}{l}\text { /rósu/ [īó.su] 'rojo' } \\
\text { /āirimát/ [a.īi.mát] 'arrimado' } \\
\text { */_\#/ }\end{array}$ \\
\hline
\end{tabular}

\subsection{Semiconsonantes}

Los fonemas semiconsonánticos se realizan como contoides y vocoides asilábicas.

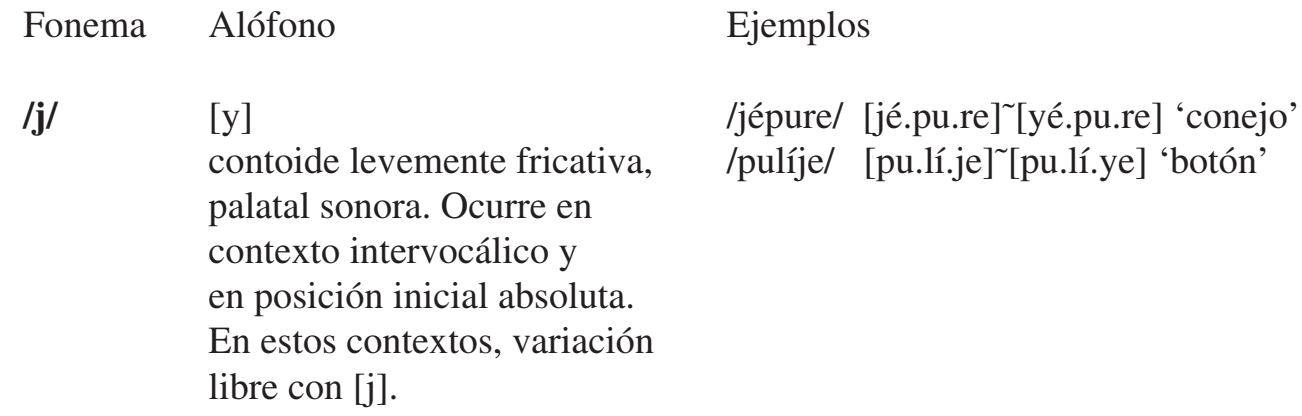


[j]

vocoide alta, cerrada,

/tjántene/ [tjáņ te.ne] [tẹénánte.ne] 'peineta'

anterior, no labializada,

asilábica. Ocurre

en los siguientes contextos:

ante una vocal tónica, en contexto

intervocálico, y en posición inicial

absoluta. Precedida de contoide,

ocurre en variación libre con [E].

[i]

/majmún/ [maĩ.mún] 'mono'

vocoide muy alta, cerrada,

anterior, no labializada,

asilábica. Sigue a una vocal tónica.

[E]

vocoide media, abierta,

anterior, no redondeada,

asilábica.

[i]

"pseudo-i”. Características

articulatorias similares a [j],

pero distinta distribución:

$\left.{ }^{[}{ }^{i}\right]$ ocurre sólo en posición

final de palabra, después

de consonante.

/w/

[?w]

contoide levemente fricativa,

velar, labializada, sonora.

Ocurre en posición inicial

absoluta y entre vocales.

En estos contextos,

variación libre con [w].

[w]

vocoide alta, posterior,

cerrada, redondeada,

asilábica. Ocurre ante

una vocal tónica, en contexto

intervocálico, y en posición inicial

absoluta. Precedida de contoide

ocurre en variación libre con [o]. /diminjáca/ [di.mi.njá.tsa] [di.mi.néá.tsa]

'madrugada'
/Éerj/ [ţ̌seri $]$ 'cielo'

/wáje/ [wá.je] [g̈wá.ye] 'oveja'

/desuswára/ [de.su.swá.ra] [de.su.sọá.ra]

'axila' 
[1].]

vocoide muy alta, cerrada, posterior, asilábica. Sigue a una vocal tónica.

[g] media, cerrada, posterior, redondeada, asilábica. /slaw/ [slay] 'flaco'

/swákra/ [sogá.kra] [swá.kra] 'suegra’

\subsection{Interpretación fonológica de algunos segmentos}

\subsubsection{Interpretación fonológica de las secuencias [tz], [dz], [t:3], [d゙]}

El principio de simetría sistémica hace preferible interpretar estas secuencias como unidades y no como complejos, ya que de otro modo serían éstas las únicas secuencias que formarían grupos de tres y cuatro consonantes en el postmargen silábico. Así, de acuerdo a esta interpretación, una palabra como ‘mucho' ([mults]) tendrá la estructura silábica frecuente en ludar /CVCC/ y no */CVCCC/.

\subsubsection{Interpretación de las vocoides [ị] [j] [u] [w]}

El funcionamiento de estos sonidos como vocales o consonantes en una lengua queda determinado por su distribución.

En ludar parece preferible interpretarlos como alófonos de las (semi)consonantes /j/ ([j] [i] $]$ y /w/ ([w] [ํ] $]$ ), pues esto permite una descripción fonotáctica más simétrica. Los argumentos son los siguientes:

a) Estos fonos tienen un funcionamiento típicamente consonántico: no ocupan la cima silábica y ocurren en el pre y postmargen silábicos.

b) Estos fonos alternan libremente con contoides en las mismas posiciones:

[jel] [yel] 'él'

[weink] [gweink] 'ojo’

c) En posición inicial absoluta no se dan grupos vocálicos aparte de los formados por algunos de estos sonidos.

d) Las estructuras silábicas que resultan de esta interpretación coinciden con las estructuras silábicas más frecuentes en ludar (ver posibilidades silábicas del ludar). 


\section{Fonotaxis}

\subsection{La sílaba}

El foco silábico siempre lo constituye una vocal.

Una palabra puede constar de:

$\begin{array}{lll}\text { Una sílaba: } & \text { /kap/ } & \text { 'cabeza' } \\ \text { Dos sílabas: } & \text { /no.rój/ } & \text { 'barro' } \\ \text { Tres sílabas: } & \text { /es.tjá.le/ } & \text { 'estrella' } \\ \text { Cuatro sílabas: } & \text { /pri.ma.vá.ra/ } & \text { 'primavera' }\end{array}$

Una vocal puede estar precedida en la misma sílaba por:

$\begin{array}{lll}\text { Una consonante: } & \text { /nor/ } & \text { 'nube' } \\ \text { Una semiconsonante: } & \text { /jo/ } & \text { 'yo' } \\ \begin{array}{l}\text { Dos consonantes: } \\ \text { Una consonante y } \\ \text { una semiconsonante: }\end{array} & \text { /praw/ } & \\ \begin{array}{l}\text { Tres consonantes: } \\ \text { Dos consonantes y } \\ \text { una semiconsonante: }\end{array} & \text { /swá.re/ } & \text { 'sol' } \\ \text { /stlí.ke/ } & \text { 'vidrio' } \\ & \text { /plwá.je/ } & \text { 'lluvia' }\end{array}$

\subsection{Distribución de los fonemas}

\subsubsection{Vocales}

Todas las vocales ocurren en posición inicial y final absoluta.

\begin{tabular}{|c|c|c|c|}
\hline 1. /ápa/ & 'agua' & 1. /zizva/ & 'día' \\
\hline 2. /estjále/ & 'estrella' & 2. /swáre/ & 'sol' \\
\hline 3. /ančápe/ & 'empieza' & 3. /mósa/ & 'abuelo' \\
\hline 4. /ínima/ & ‘corazón’ & 4. /sínịiri/ & 'cadena' \\
\hline 5. /iste/ & 'éste' & 5. $/ \mathrm{kij} /$ & 'que' \\
\hline 6. /orjés/ & 'arroz' & 6. /mámo/ & 'madre' \\
\hline 7. /úmbra/ & 'sombra' & 7. $/ \mathrm{tu} /$ & 'tú' \\
\hline
\end{tabular}

\subsubsection{Consonantes y semiconsonantes}

5.2.2.1. Una consonante

En posición inicial de palabra ocurren todas las consonantes, excepto la vibrante simple /r/.

$\begin{array}{lll}\text { /pV/: } & \text { /púla/ } & \text { 'pene' } \\ \text { /bV/: } & \text { /burík/ } & \text { 'ombligo' }\end{array}$




\begin{tabular}{|c|c|c|}
\hline$/ \mathrm{tV} /:$ & /táta/ & 'padre' \\
\hline /dV/: & /dint/ & 'diente' \\
\hline /kV/: & /kaús/ & 'cucharón' \\
\hline /gV/: & /gol/ & ‘vacío' \\
\hline /fV/: & /fum/ & 'humo' \\
\hline /vV/: & /vìlint/ & 'valiente' \\
\hline /sV/: & /sókra/ & 'suegro' \\
\hline /zV/: & /záma/ & 'sopa' \\
\hline /sV/: & /sap/ & 'bolsillo' \\
\hline /ž V/: & /žinžíl/ & 'encía' \\
\hline /cV/: & /car/ & 'gobierno' \\
\hline /दु V/: & /Z̧áne/ & 'ceja' \\
\hline /: V/: & /č́epéla/ & 'zapato' \\
\hline$/ \mathrm{j} \mathrm{V} / \mathrm{:}$ & jjinér/ & 'yerno' \\
\hline$/ \mathrm{mV} /:$ & /mucis/ & 'mucus' \\
\hline /nV/: & /nepót/ & 'nieto' \\
\hline /lV/: & /lumína/ & 'luz' \\
\hline$* / \mathrm{rV} /$ & & \\
\hline /IV/: & rrosu/ & 'rojo' \\
\hline
\end{tabular}

En contexto intervocálico ocurren todas las consonantes. En este contexto, toda consonante forma sílaba con la vocal que le sigue:

\begin{tabular}{|c|c|c|}
\hline /V.pV/: & /Čá.pə/ & 'cebolla' \\
\hline /V.bV/: & /dó.ba/ & 'hora' \\
\hline /V.tV/: & /pì.tura/ & 'cama' \\
\hline /V.dV/: & /da.da.raš/ & 'pantalones \\
\hline /V.kV/: & /te.kút/ & 'callado' \\
\hline /V.gV/: & /bo.gát/ & 'rico' \\
\hline /V.fV/: & /a.fár/ & 'afuera' \\
\hline /V.vV/: & /no.sá.va/ & 'muela' \\
\hline /V.sV/: & /ma.nú.sa/ & 'mango' \\
\hline /V.zV/: & /na.ze.vít/ & 'resfriado' \\
\hline /V.sV/: & /ba.sár/ & 'besar' \\
\hline /V.ž V/: & /a.žú.ta/ & 'ayuda' \\
\hline /V.cV/: & /ku.cət/ & 'cuchillo' \\
\hline /V.zV/: & /fú.z̧e/ & 'arranca' \\
\hline /V V/: & /pi.č́ór/ & 'pie' \\
\hline /V.j V/: & /pa.la.já.ne/ & 'tomate' \\
\hline /V.mV/: & /ki.ma.sa/ & 'camisa' \\
\hline /V.nV/: & /mi.ní.ka/ & 'manga' \\
\hline /V.IV/: & /tì.líi// & 'mentón' \\
\hline /V.rV/: & /u.rit/ & 'feo' \\
\hline /V.ĪV/: & /a.Īi.mát/ & 'arrimado' \\
\hline
\end{tabular}


En posición final de palabra ocurren las siguientes consonantes:

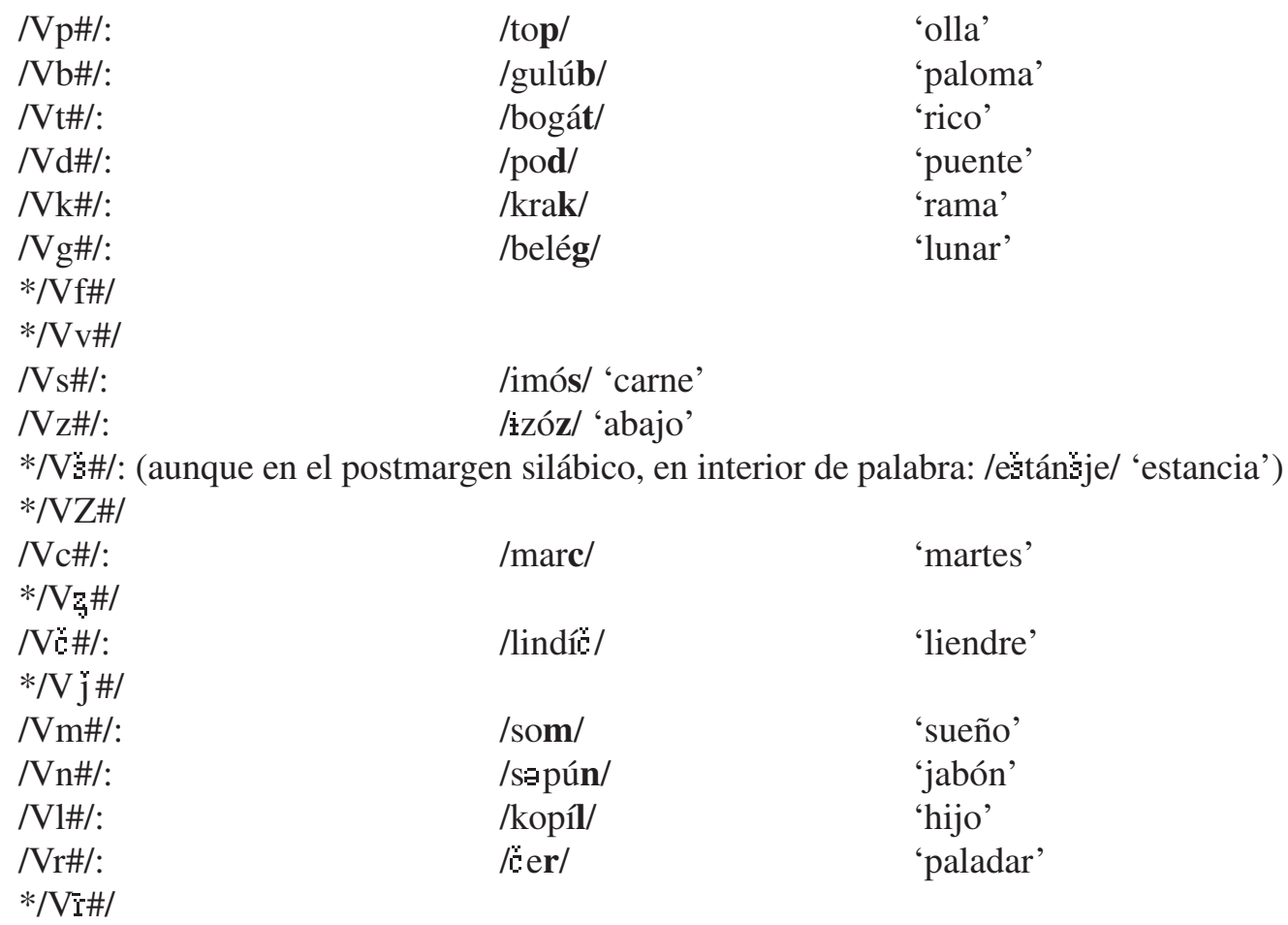

\subsubsection{Una semiconsonante}

En posición inicial de palabra ocurren las dos semiconsonantes:

$\begin{array}{lll}\text { /\#jV/: } & \text { /já.le/ } & \text { 'ellas' } \\ \text { /\#wV/: } & \text { /wá.je/ } & \text { 'oveja' }\end{array}$

En contexto intervocálico ocurren las dos semiconsonantes. En este contexto, forman sílaba con la vocal siguiente.
/V.jV/:
/mu.já.re/
'mujer'
/V.wV/:
/sa.wá.dje/
'mariposa'

En posición final de palabra ocurren las dos semiconsonantes:
/j\#/:
$/$ doj/
'dos'
/w\#/:
/Îw/
'malo'

5.2.2.3. Una consonante más una semiconsonante 
En posición inicial de palabra ocurren los siguientes grupos:

$/ \mathrm{p}, \mathrm{b}, \mathrm{t}, \mathrm{d}, \mathrm{k}, \mathrm{g}, \mathrm{v}, \mathrm{m}, \mathrm{n}, \mathrm{l}, \overline{\mathrm{I}} /+/ \mathrm{j} /$

\begin{tabular}{|c|c|}
\hline /pjV/: & /pjápene/ \\
\hline /bjV/: & /bja/ \\
\hline$/ \mathrm{tjV} /:$ & /tjántene/ \\
\hline /djV/: & /dja/ \\
\hline$/ \mathrm{kjV} / \mathrm{:}$ & /kjáma/ \\
\hline /gjV/: & /gjórge/ \\
\hline /vjV/: & /vjárde/ \\
\hline$/ \mathrm{mjV} /:$ & /mjáre/ \\
\hline /njV/: & /njekurátu/ \\
\hline /ljV/: & /ljag/ \\
\hline 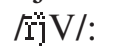 & /Îjáce/ \\
\hline
\end{tabular}

'pescado'

'bebió'

'peineta'

'dio'

'llamar'

'Jorge'

'verde'

'miel'

'demonio'

'atar'

'frío'

/p, b, t, d, k, s, z, m, n, $\overline{\mathrm{I}} /+$ /w/

/pwV/:

/pwála/

'costado'

/bwV/:

/bwáva/

'bombilla'

/twV/:

/twála/

/dwV/:

/dwavesíc/

'toalla'

$/ \mathrm{kwV} /$ :

/kwádə/

'veinte'

/swV/:

/swákra/

'cola'

/zwV/:

/zwáka/

'suegra'

$/ \mathrm{mwV} /$ :

/mwále/

/nwV/:

/nwávä/

/I $\mathrm{WV} /$ :

/Îátē/

'bailar'

'moler'

'nueve'

'rueda'

En contexto intervocálico, la semiconsonante forma sílaba con la consonante precedente y la vocal siguiente. Algunos de los grupos más frecuentes en este contexto son los siguientes:

$/ \mathrm{p}, \mathrm{t}, \mathrm{d}, \mathrm{f}, \mathrm{v}, \mathrm{s}, \mathrm{n}, \mathrm{r}, \mathrm{l} / \mathrm{+} / \mathrm{j} /$

N.pjV/

/V.tjV/

/V.djV/

/V.fjV/

/V.vjV/

/V.sjV/

/V.njV/

/V.rjV/

/V.ljV/

$/ \mathrm{p}, \mathrm{t}, \mathrm{d}, \mathrm{s}, \mathrm{m}, \mathrm{n}, \mathrm{r} /+\mathrm{w} /$

/V.pwV/

/V.twV/

/skli.pjás.te/
/kár.tja/
/ku.ku.djás.te/
/ka.fjá.va/
/po.vjás.ta/
/mi.sjá.ko/
/i.njél/
/má.rja/
/za.pa.ljás.te/

'brilla'

'carta'

'se sana'

'café'

'cuento'

'ratón'

'anillo'

'mar'

'fuma'

/ne.pwá.ta/

/ser.va.twá.ra/ 'nieta'

'virgen' 


$\begin{array}{lll}\text { /V.dwV/ } & \text { /pa.dwî́/ } & \text { 'piojo' } \\ \text { /V.swV/ } & \text { /de.su.swá.ra/ } & \text { 'axila' } \\ \text { /V.mwV/ } & \text { /pul.mwá.ne/ } & \text { 'pulmones' } \\ \text { /V.nwV/ } & \text { /re.nwíc/ } & \text { 'riñones' } \\ \text { /V.rwV/ } & \text { /ku.ku.rwá.de/ } & \text { 'torta frita' }\end{array}$

En posición final sólo ocurren grupos con la semiconsonante /j/. Algunos de estos grupos son los siguientes:

$\begin{array}{lll}\text { /V.bj/ } & \text { /bubj/ } & \text { 'granos' } \\ \text { /V.tj/ } & / \text { mutj/ } & \text { 'mudo' } \\ \text { /V.nj/ } & / \text { lunj/ } & \text { 'lunes' } \\ \text { /V.rj/ } & / \text { / erj/ } & \text { 'cielo' }\end{array}$

5.2.2.4. Una semiconsonante más una consonante

Este grupo no ocurre en posición inicial de palabra.

En contexto intervocálico la semiconsonante pertenece a la sílaba de la vocal precedente y la consonante a la sílaba de la vocal siguiente. Algunas secuencias frecuentes en esta posición son las siguientes:

$$
/ \mathrm{j} /+/ \mathrm{s}, \mathrm{c}, \mathrm{m}, \mathrm{r} /
$$

/Vj.s/

/trej.Sic/

'treinta'

/Vj.c/

/baj.ç1/

'niño'

/Vj.m/

/maj.mún/

'mono'

/Vj.r/

/baj.rá.ku/

'bandera'

$/ \mathrm{w} /+/ \mathrm{t}, \mathrm{d} /$

/Vw.tV/

/jéw.tin/

'barato'

/Vw.dV/

/láw.de/

'alabar'

Ejemplos de esta secuencia en posición final de palabra son las siguientes:
$/ \mathrm{VjC \# /}$
/ $\mathrm{WWCH}$
/wejk/
/kowt/
'ojo'
'maduro'

\subsubsection{Dos consonantes}

En posición inicial de palabra, los grupos más frecuentes son los siguientes:

$/ \mathrm{p}, \mathrm{t}, \mathrm{d}, \mathrm{k}, \mathrm{g}, \mathrm{f} / \mathrm{+} / \mathrm{r} /$ :

$/ \mathrm{prV} /$ :

$/ \operatorname{trV} /$ :

/praw/

'tierra'

$/ \mathrm{drV} /$ :

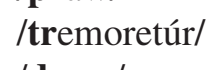

'temblar'

/drum/

'camino' 


\begin{tabular}{|c|c|c|}
\hline $\begin{array}{l}\text { /krV/: } \\
/ \mathrm{grV} /: \\
/ \mathrm{frV} /:\end{array}$ & $\begin{array}{l}\text { /krak/ } \\
\text { /grimma/ } \\
\text { /frá.te/ }\end{array}$ & $\begin{array}{l}\text { 'rama' } \\
\text { 'pulsera' } \\
\text { 'hermano' }\end{array}$ \\
\hline $\begin{array}{l}/ \mathrm{p}, \mathrm{b}, \mathrm{g} /+/ \mathrm{l} /: \\
/ \mathrm{plV} / \\
/ \mathrm{blV} / \\
/ \mathrm{glV} /\end{array}$ & $\begin{array}{l}\text { /plánt:a/ } \\
\text { /blastemát/ } \\
\text { /glonc/ }\end{array}$ & $\begin{array}{l}\text { 'planta' } \\
\text { 'maldito' } \\
\text { 'bala' }\end{array}$ \\
\hline $\begin{array}{l}\text { /s/ + /p, t, k, 1/: } \\
/ \mathrm{spV} /: \\
/ \mathrm{stV} /: \\
\text { /skV/: } \\
\text { /slV/: }\end{array}$ & $\begin{array}{l}\text { /sperját/ } \\
\text { /sting/ } \\
\text { /skam/ } \\
\text { /slaw/ }\end{array}$ & $\begin{array}{l}\text { 'asustado' } \\
\text { 'izquierdo' } \\
\text { 'silla' } \\
\text { 'flaco' }\end{array}$ \\
\hline
\end{tabular}

En contexto intervocálico, hay que distinguir dos casos:

a) la primera consonante pertenece a la sílaba de la vocal precedente y la segunda a la sílaba de la vocal siguiente y

b) ambas consonantes pertenecen a la sílaba de la vocal siguiente.

Algunas secuencias frecuentes para a) son las siguientes:

$/ \mathrm{n} /+/ \mathrm{t}, \mathrm{d}, \mathrm{k}, \mathrm{g}, \stackrel{\mathrm{c}}{\mathrm{b}}, \mathrm{c}, \mathrm{j}, \ddot{\mathrm{z}} /$

\begin{tabular}{|c|c|c|}
\hline /Vn.tV/ & /un.tú.re/ & 'grasa' \\
\hline /Vn.dV/ & /lin.dî́: / & 'liendre' \\
\hline /Vn.kV/ & /mun.ká.re/ & 'comer' \\
\hline /Vn.gV/ & /lín.gu.re/ & 'cuchara' \\
\hline /Vn.ťn/ & /min.č́néne/ & 'mentira' \\
\hline /Vn.cV/ & /cin.cár/ & 'mosquito' \\
\hline /Vn.j V/ & /sin.jú.ri/ & 'cadena' \\
\hline / Vn.z̄ V/ & /žin.žíl & 'encía' \\
\hline \multicolumn{3}{|c|}{$/ \mathrm{r} / \mathrm{t} / \mathrm{p}, \mathrm{b}, \mathrm{t}, \mathrm{d}, \mathrm{k}, \mathrm{g}, \mathrm{z}, \mathrm{c}, \mathrm{m}, \mathrm{n} /$} \\
\hline /Vr.pV/: & /kir.pa/ & ‘pañuelo’ \\
\hline /Vr.bV/: & /kar.bú.ne/ & ‘carbón’ \\
\hline /Vr.tV/: & /búr.ta/ & 'vientre' \\
\hline /Vr.dV/: & /vjár.de/ & 'verde' \\
\hline /Vr.kV/: & /fúr.ka/ & 'poste de la carpa' \\
\hline /Vr.gV/: & /gar.ga.ú.ne/ & 'abeja' \\
\hline /Vr.zV/: & /var.ze/ & 'repollo' \\
\hline /Vr.c. V/: & /por.čááva/ & 'chancha' \\
\hline /Vr.mV/: & /mír.mīn.te/ & 'cementerio' \\
\hline /Vr.nV/: & /fur.ní.ka/ & 'hormiga' \\
\hline
\end{tabular}


/s/ + /t, d, k, m/

/Vs.tV/:

/as.túk/

/Vs.dV/:

/gás.da/

/Vs.kV/:

/be.lús.ka/

/Vs.mV/:

/dus.mán/

'plumón'

'dueño'

'tenedor'

'enemigo'

/l/ + /t, d, k, s, v, ò

/Vl.tV/:

/al.téw/

'tuyo'

/ses.kál.də/

'se baña'

Vl.dVI

/kil.kijj/

'talón'

/Vl.sV/

/púl.so/

/Vl.vV/

/al.vós.tru/

/Vlí̃ V/

/dúlợe/

'poste de la carpa'

'vuestro'

'dulce'

$/ \mathrm{m} /+/ \mathrm{p}, \mathrm{b} /$

/Vm.pV/

/lím.pi.de/

/lím.ba/

'limpio'

'lengua'

Algunas secuencias frecuentes para b) son las siguientes:

$/ \mathrm{p}, \mathrm{t}, \mathrm{k}, \mathrm{g}, \mathrm{f}, \mathrm{v} / \mathrm{+} / \mathrm{r} /$

/V.prV/

/V.trV/

/V.krV/

/V.grV/

/V.frV/

/V.vrV/

/f, $\mathrm{v} /+/ \mathrm{l} /$

/V.flV/

/V.vlV/

/a.prín/
/mí.tra/
/lá.kri.ma/
/njé.gra/
/ka.frín/
/i.vrík/

/sú.fla/

/o.vlín.de/ 'enciende'

(nombre propio)

'lágrima'

'negro'

'clavo'

'fuera'

'soplar'

'espejo'

Algunos grupos frecuentes en posición final de palabra son los siguientes:

$/ \mathrm{n} / \mathrm{t} / \mathrm{t}, \mathrm{d}, \mathrm{k}, \mathrm{g}, \mathrm{s}, \mathrm{c}, \mathrm{c} /$

/Vnt\#/

/Vnd\#/

/Vnk\#/

/Vng\#/

/Vns\#/

/Vnc\#/

/Vnơ̈\#/

/vint/
/blind/
/mì.ník/
/a.î́ng/
/as.kúns/
/njanc/
/ôinins/

'viento'

'blando'

'comer'

'tirar'

'esconder'

'no-gitanos'

'cinco' 
$/ \mathrm{r} /+/ \mathrm{t}, \mathrm{d}, \mathrm{k}, \mathrm{g}, \mathrm{s}, \mathrm{v} /$

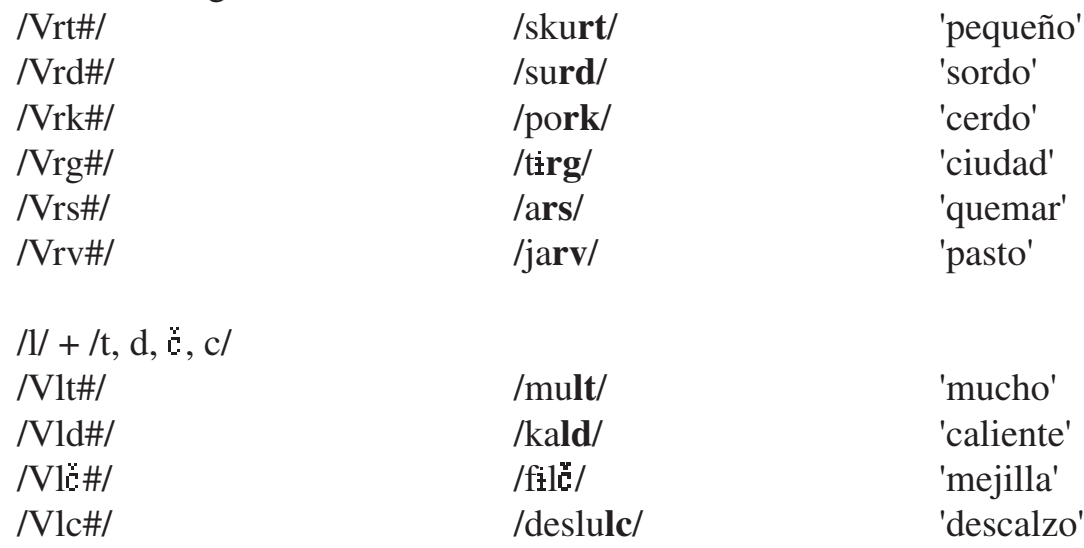

\subsubsection{Tres consonantes}

Ejemplos de secuencias de tres consonantes en posición inicial de palabra son los siguientes:

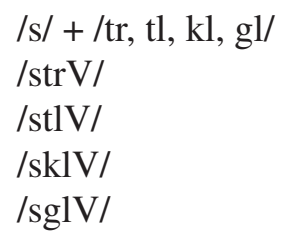

$\begin{array}{ll}\text { /strú.gur/ } & \text { 'uva' } \\ \text { /stlí.ke/ } & \text { 'vidrio' } \\ \text { /skli.pjás.te/ } & \text { 'brilla' } \\ \text { /sglo.bo.zìt/ } & \text { 'destruyó' }\end{array}$

En contexto intervocálico, la primera consonante pertenece a la sílaba de la vocal precedente y las otras dos a la sílaba de la vocal siguiente. Algunas secuencias que ocurren en esta posición son las siguientes:

/n/+ /tr, dr, gr,/

$\begin{array}{lll}\text { /Vn.trV/ } & \text { /in.ón.tru/ } & \text { 'dentro' } \\ \text { /Vn.drV/ } & \text { /mf́n.dru/ } & \text { 'hermoso' } \\ \text { /Vn.grV/ } & \text { /an.gro.pát/ } & \text { 'enterrado' }\end{array}$

$/ \mathrm{m} /+/ \mathrm{br}, \mathrm{bl} /$

/Vm.brV/

/úm.bra/ 'sombra'

/Vm.blV/

/um.blá/

'caminaban'

/s/+ /tr, kl/

/Vs.trV/

/nós.tru/ 'nuestro'

/Vs.klV/

/es.kli.pe.túr/

'rayo'

Esta secuencia no ocurre en posición final de palabra. 
5.2.2. Dos consonantes y una semiconsonante

Ejemplos de secuencias de dos consonantes y una semiconsonante en posición inicial de palabra son los siguientes:

$\begin{array}{lll}\text { /kr, gr, sk/ + /j/ } & \\ / \mathrm{krjV} / & \text { /krjás.ta/ } & \text { 'cresta' } \\ \text { /grjV/ } & \text { /grjew/ } & \text { 'pesado' } \\ \text { /skjV/ } & \text { 'cojo' } \\ & & \\ \text { /plop/ } \mathrm{st}, \mathrm{gr} /+ \text { /w/ } & \\ \text { /plwV/ } & & \\ \text { /stwV/ } & \text { /plwá.je/ } & \text { 'lluvia' } \\ \text { /grwV/ } & \text { /stwéna/ } & \text { (nombre propio) } \\ \text { /grwá.pe/ } & \text { 'tumba' }\end{array}$

En contexto intervocálico hay que distinguir dos casos: a) la primera consonante pertenece a la sílaba de la vocal precedente y la otra secuencia pertenece a la sílaba de la vocal siguiente (/VC.CScV/) y b) toda la secuencia pertenece a la sílaba de la vocal siguiente (/V.CCScV/).

Algunas secuencias que pertenecen al grupo a) son las siguientes:

/nt, sm, rp, rb, rt, nd/ + /j/

$\begin{array}{lll}\text { /Vn.tjV/ } & \text { /frún.tja/ } & \text { 'frente' } \\ \text { /Vs.mjV/ } & \text { /is.mjá.ne/ } & \text { 'calzoncillos' } \\ \text { /Vr.pjV/ } & \text { /kir.pjás.te/ } & \text { 'coser' } \\ \text { /Vr.bjV/ } & \text { /bor.bjás.te/ } & \text { 'hablar' } \\ \text { /Vr.tjV/ } & \text { /kár.tja/ } & \text { 'carta' } \\ \text { /Vn.djV/ } & \text { /gan.djá/ } & \text { 'creía' } \\ & & \\ \text { /lm/ } & + \text { /w/ } & \\ \text { /Vl.mwV/ } & \text { /pul.mwá.ne/ } & \text { 'pulmones' }\end{array}$

Algunas secuencias que pertenecen al grupo b) son las siguientes:

/vl, $\mathrm{kr} /+/ \mathrm{j} /$

$\begin{array}{lll}\text { /V.vljV/ } & \text { /do.vlják/ } & \text { 'zapallo' } \\ \text { /V.krjV/ } & \text { /lu.krjás.te/ } & \text { 'trabajar' }\end{array}$

Esta secuencia no ocurre en posición final de palabra. 


\section{Posibilidades silábicas del ludar}

$\begin{array}{lll}\text { 1. V } & \text { /o.vlín.de/ } & \text { espejo } \\ \text { 2. VC } & \text { /es.tjá.le/ } & \text { estrella } \\ \text { VSc } & \text { /ow/ } & \text { huevo } \\ \text { 3. VCC } & \text { /urs/ } & \text { pozo } \\ \text { VScC } & \text { /owt/ } & \text { ocho } \\ \text { 4. CV } & \text { /no.rój/ } & \text { barro } \\ \text { ScV } & \text { /ja/ } & \text { ella } \\ \text { 5. CVC } & \text { /nor/ } & \text { nube } \\ \text { CVSc } & \text { /no.rój/ } & \text { barro } \\ \text { ScVC } & \text { /jár.na/ } & \text { invierno } \\ \text { ScVSc } & \text { /jej/ } & \text { ellos } \\ \text { 6. CVCC } & \text { /pírg/ } & \text { pelo } \\ \text { CVScC } & \text { /majn.dá.te/ } & \text { apurado } \\ \text { CVCSc } & \text { /búbj/ } & \text { granos } \\ \text { ScVScC } & \text { /wejk/ } & \text { ojo } \\ \text { 7. CCV } & \text { /frá.te/ } & \text { hermano } \\ \text { CScV } & \text { /swá.re/ } & \text { sol } \\ \text { 8. CCVC } & \text { /frán.te/ } & \text { frente } \\ \text { CCVSc } & \text { /praw/ } & \text { tierra } \\ \text { CScVC } & \text { /pjás.te/ } & \text { pescado } \\ \text { CScVSc } & \text { /nwáw.tje/ } & \text { noche } \\ \text { 9. CCVCC } & \text { /glonc/ } & \text { bala } \\ \text { CScVCC } & \text { /an.mjérs/ } & \text { fui } \\ \text { CScVScC } & \text { /u.rjéjk/ } & \text { oreja } \\ \text { 10. CCCV } & \text { /skli.pjás.te/ } & \text { brilla } \\ \text { CCScV } & \text { /plwá.je/ } & \text { lluvia } \\ \text { 11. CCCVC } & \text { /sprág.nik/ } & \text { promesa } \\ \text { CCScVC } & \text { /krjás.ta/ } & \text { cresta } \\ \text { CCScVSc } & \text { /grjew/ } & \text { pesado } \\ & & \end{array}$

Hemos encontrado los siguientes casos de fluctuación de fonemas:
1. $/ a / \% / \mathrm{a} /$
2. $\mid a / / \mathrm{e} /$
3. $/ \mathbf{i} / / \mathrm{i} /$
4. $/ 2 / \% / \mathrm{u} /$
5. $/ \mathrm{o} / \% / \mathrm{u} /$
6. $/ \mathrm{j} / / \mathrm{i} /$
7. $/ \mathrm{v} / / \mathrm{b} /$
8. $/ \mathrm{z} / \% / \mathrm{s} /$
9. $/ \check{2} / \sim \mathrm{j} /$

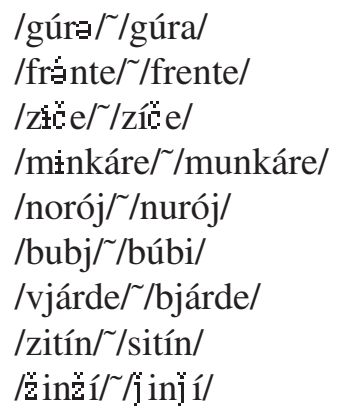

'cara, boca'

'frente'

'dice'

'comer'

'barro'

'granos'

'verde'

'aceite'

'encía' 


\section{Breve comparación del inventario de vocales y consonantes presentado aquí con los que se contienen en los estudios fonológicos clásicos de la lengua rumana}

Aunque no constituye el foco de este artículo, me ha parecido interesante comparar el inventario de fonemas del ludar con los que aparecen en las descripciones fonológicas clásicas de la lengua rumana. Así, pues, comparé el inventario de vocales con los que presenta Petrovic, Rossetti y Avram (reseñados en Avram 1987). Esta comparación me permitió establecer que nuestra interpretación coincide con la de Rossetti y Avram (estos autores distinguen siete vocales fonológicas) y se distancia de la interpretación de Petrovic (para este autor el inventario fonológico del rumano tiene sólo 5 vocales: /ì, ə, a, o, u/). La comparación del inventario de consonantes arroja resultados similares: nuestro inventario de consonantes es semejante al que se presenta en Avram y Rossetti y se distancia de la interpretación de Petrovic (1956) (este autor distingue 72 fonemas consonánticos). ${ }^{5}$

\section{Transcripción fonémica de un relato en ludar (traducción libre)}

da mult dummezéw umblá pa zos

antiguamente Dios andaba caminando

umblá deskúlc i plin de bárbe

andaba descalzo y lleno de barba

atúnča aplekát la o káse muj bogáte se čáje meláj

entonces llegó a una casa muy hermosa a pedir pan

i ş̣̂rva dẹ ač̣ía kúm irjá bogáta alát un krodiốéj de maláj

y la serba ${ }^{6}$ de ahí como era rica tomó un pedazo de pan

i le ar̄unkát ku pičóru

y lo tiró con el pie

ku pičóru dja la maláju

con el pie le dio el pan

dummezéw alát maláju y sa fakút la un ĩančít

Dios tomó el pan y se fue a un ranchito

ačía ìn T̄anớítola irjá o sérva muj seráke

ahí en el ranchito había una serba muy pobre

ki avjá un kopíl plin de búbj

que tenía un niño lleno de granos 
plin de bubj pə gúre

lleno de granos en la cara

kopilásu plinṇ̂á i plìnj̣á

el niño lloraba y lloraba

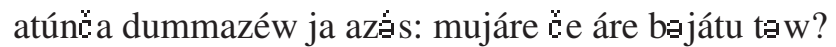

entonces Dios dijo: mujer ¿qué tiene tu hijo?

nu stiw azês, náno, nu stiw č̣e áre, pero pliłnj̄e

no sé dijo, padrino, no sé que tiene, pero llora.

bwíte č̣e áre pa gúra

mira que tiene en la cara

bueno, ja azés dummezéw inklisáste órnu

bueno, dijo Dios, calienta el horno

i bágìle in óntru ki prjaw sa le minink

y ponlo dentro que quiero comérmelo

sə̉rva apríns órnu

la serba prendió el horno

i kịndə sapús bíne r̄ósu ja bəgáte bəjátu in óntru

y cuando se puso muy rojo puso el hijo dentro

entonse sérva múma la bajátuli plinị̂a i plìnịá

entonces la serba, la madre del niño, lloraba y lloraba

porque gandjá ki bajátu sa frị̂á

porque creía que el niño se quemaba

atúnç̣ a dummazéw adát ku bastónu ìn orn

entonces Dios dio con el bastón en el horno

i órnu sa stins

y el horno se apagó

i azき́s: bueno, mujáre áde bəjátu se le mininink

y dijo: bueno, mujer, trae el niño para comérmelo

i la mujáre skwáte bajátu dən órnu

y la mujer saca al niño del horno 
i bajátu irjá likwít de bubj

y el niño estaba sanado de los granos

minndru minndru irjá bəjátu

hermoso hermoso estaba el niño

atúnça sảrva seráke ja fakút o munkáre la dummazéw

entonces la serba pobre hizo comida para Dios

ja dat kafjá, məláj, ja pus mása

le dio queso, café, pan, puso la mesa

despwés ja dat ápə sə sa spjále pa piốwáre

después le dio agua para que se lavara los pies

ja dat tjántene sa sa tjántene

le dio peineta para que se peinara

atúnč̀a dummazéw ja azés: ¿̌e prjaj tu mujáre?

entonces Dios dijo: ¿qué quieres tú, mujer?

jo prjaw lúkru la cigánu mew i o kása ka lúmja

yo quiero trabajo para mi gitano y una casa como la gente

i dummazéw ku bastónu afakút trej kruỡ

y Dios con el bastón hizo tres cruces

i adát č̣egó abrút la mujárja áste

y dio todo lo que quería la mujer ésta

i pa bogáte sglobəzit o torménte ki ja Īut twáte twáte kase. y a la rica le envió una tormenta que rompió toda toda su casa.

\section{Notas}

1. Este artículo contiene una versión simplificada de mi tesis de Magister en Lingüística "Descripción fonológica del ludar, lengua hablada por un grupo gitano de Argentina", dirigida por el Dr. Adalberto Salas. Los datos para la elaboración de este trabajo los recogí junto a mi colega Álvaro González en la ciudad de Buenos Aires.

2. Algunos de los países americanos en los cuales existen gitanos ludar son Argentina, Brasil y Chile; Rumania, Yugoslavia y Hungría son algunos de los países europeos en los cuales está presente este grupo.

3. Para una descripción etnográfica más detallada puede consultarse "Gitanos: Algunos antecedentes etnográficos” (Salamanca 1999) y “Gitanos de Chile: Un acercamiento etnolingüístico” (Salamanca y González 1999). 
4. No disponemos de datos confiables que indiquen el número de gitanos ludar presentes hoy en Argentina. Lo que resulta claro de nuestra observación in situ y del testimonio de los propios gitanos es que éstos constituyen una minoría respecto de los gitanos rom.

5. La gran cantidad de fonemas consonánticos que entrega Petrovic se explica porque este lingüista "classified [the consonantal phonemes] in four categories: neutral (18), palatalized (21), rounded (19), and palatalized-rounded (14)." (Avram 1987, 91). En nuestro análisis, el problema de los fonemas consonánticos palatalizados lo hemos resuelto considerando que la palatalización no es un rasgo de una serie de fonemas consonánticos, sino una realización alofónica de la semiconsonante /j/. Esta solución, que coincide con la de Avram 1976, pensamos que es fonológicamente plausible por cuanto respeta los principios de economía sistémica y de simetría de pauta, sin perder por ello las necesarias distinciones fonológicas.

6. Habitante de Serbia, región donde se sitúa el cuento.

\section{Bibliografía}

Avram, A. 1987. "Rumanian and General Phonetics and Phonemics". Revue Rumaine de Linguistique. Tome XXIII: 71-97.

Echeverría, M. 1964. "Descripción fonológica del mapuche actual”. Boletín de Filología XVI. Santiago: 13-59.

Grant, A. 1994. Romani: a conspectus. Notes on Romani Language and Linguistics. Departament of Modern Language. University of Bradford. England.

Hancock, I. 1993. Grammar of Vlax Romani. Texas: Romanestan Publications.

Ivantts, A. 1974. "Los gitanos y el mundo moderno”. Correo de la Unesco: 4-10.

Pike, K. 1947. Phonemics. Ann Arbor: The University of Michigan Press.

Réger, Z. 1995. "The language of Gypsies in Hungary: an overview of research". Journal of Sociology of Language: 79-91.

Rishi, W. 1981. "Brief Grammar of Romani Language”. Language Department. Punjab. Delhi.

Salamanca, G. 1999. “Gitanos: Algunos antecedentes etnográficos”. Sociedad Hoy. Universidad de Concepción. Chile.

Salamanca, G. y A. González 1999. “Gitanos de Chile: Un acercamiento etnolingüístico”. Atenea. Universidad de Concepción. Chile.

Vaux de F., F. 1984. "De los países del indo al mundo occidental". El Correo de la Unesco. Año XXXVII: 5-7.

Van der Voort, H. 1996. “The Romani dialect(s) of the finnish Gypsies”. Amsterdam: Institute for General Linguistics. 